\title{
Recent Research Trends of Plant Disease Detection
}

\author{
Vaibhavi S. Bharwad ${ }^{1}$, Kruti J. Dangarwala ${ }^{2}$ \\ ${ }^{1}$ Department of Information Technology, SVM Institute of Technology, Bharuch 392-001, Gujarat, India \\ ${ }^{2}$ Department of Computer Engineering / IT, SVM Institute of Technology, Bharuch 392-001, Gujarat, India
}

\begin{abstract}
Diagnosis of plant disease is a task of identifying the disease in the leaf or fruit or vegetable. About $42 \%$ of the world's agriculture harvest is destroyed yearly by disease and pest. However, losses of harvest can be minimized and specific treatments can be applied if plant diseases are correctly identified early. Manual identification of disease in the plant is not only time consuming but also does not give accurate result. So, providing fast, automatic and accurate solution using image processing techniques can be a good realistic significance. Automatic identification of diseases using image processing techniques can be done by using five methods like Image Acquisition, Image Pre- processing, image segmentation, Feature extraction and classification. Over last few years, many research works are carrying out in this area. This paper provides a brief overview on methodology. Further, paper reviews recent research trends to identifying disease in the plant which is based on image processing techniques.
\end{abstract}

Keywords: Leaf disease detection, plant disease, fruit disease, Disease classification

\section{Introduction}

In India, the agricultural sector gets a vital position in the overall economy of the country. Agriculture sector provides livelihood to 65 to 70 percent of the total population. Many big and small scale industries are depend on agriculture sector for their raw-material lie sugar factory, cotton and jute textile industries, food industries, pharmaceutical industry or many more. All industries need good Quality raw-material. So, Research in agricultural sector is aimed towards increase of productivity and quality.

Plant disease is one of the crucial causes that reduces quantity and degrades quality of the agricultural production [1]. Disease is an impairment of health or a condition of abnormal functioning. Plant diseases are usually caused by bacteria, viruses and fungi. The occurrence of plant diseases also depend on environmental condition. Disease requires careful diagnosis and handling at right time to protect the plant from heavy losses. Disease can be found in different parts of the plant like fruit, leaves, vegetable, and stem. Detecting disease in a plant plays a major role in the field of agriculture [2]. Monitoring of health and detection of disease in plants and trees is critical for sustainable agriculture.

Farmers detect disease through their observation of naked eyes, which is very difficult and require continuous monitoring of the plant. There is also possibility of inaccurate result and this method is expensive for large farms. In some places, farmer may have to go long distance to contact experts, which is very expensive and time consuming. Every time it is not feasible to call experts for diagnosis. So, Automatic detection of plant diseases is an important research topic as it may prove benefits in monitoring large field of crops, and thus automatically detect diseases from symptoms that appear on plant. Thus automatic detection of plant disease with the help of image processing technique provides more accurate result which help in disease management.

Structuring of remaining paper is as follows. Section II provides overview methodology which is used for plant disease detection. Section III focuses on recent research trends in plant disease detection. Furthermore, Section IV concludes the paper.

\section{Methodology}

There are five main steps for the detection of plant disease as Image acquisition, Image Pre-processing, Image segmentation, Feature extraction and Classification The processing scheme consists of image acquisition through digital camera or scanner, image pre-processing includes image enhancement, image segmentation where the affected and useful area are segmented, feature extraction and classification which classify disease [3]. Fig.1 shows the framework of system.

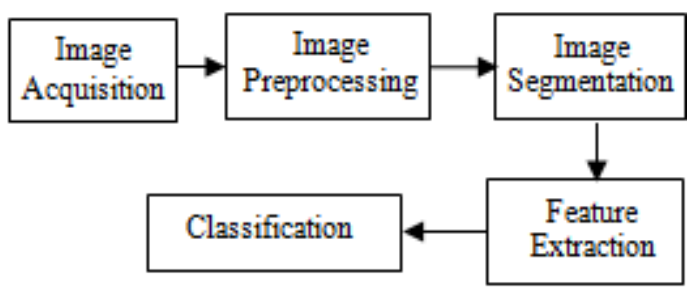

Figure 1: Framwork for plant disease detection

\section{A. Image Acquisition:}

In image processing, image acquisition can be defined as the retrieving of an image using some sources, which is a first step for any vision system. Usually hardware based sources are used for image retrieval like camera and scanner. Types of hardware which is used for image retrieval is very important. The image, which is retrieval using some source is completely unprocessed image. One of the main goals of this step is to have source of input. 


\section{International Journal of Science and Research (IJSR) \\ ISSN (Online): 2319-7064 \\ Index Copernicus Value (2013): 6.14 | Impact Factor (2014): 5.611}

\section{B. Image pre-processing:}

Image pre-processing is also known as image restoration. Image pre-processing is the lowest level of abstraction whose aim is to improve the image data that suppress undesired distortions as well as enhances some image Features which is important for further processing and analysis task [3].Image pre-processing include image Resampling, image enhancement and noise removal. In image re-sampling dimensions of the pixel is change. In image enhancement brightness of the image is increased. In image, noise is the result of errors in image acquisition process which decrease the quality of image so in noise removal process reduction of noise is done.

\section{Image Segmentation:}

In image processing, image segmentation is the process of dividing or clustering the image in to number of parts containing each pixel with same value. Image segmentation is also done through feature based clustering [3]. In the current work, the purpose of segmentation is to identify regions in the image that are likely to qualify as diseased regions [1]. Numbers of techniques are used for the segmentation process. The most frequently used technique for the segmentation is $\mathrm{K}$-means clustering.

\section{Feature Extraction}

Main purpose of this step is to find and extract features that can be used to determine the meaning of a given image. Image features extraction includes color, texture and shape in image processing which is a most common visual feature [3].

\section{- Color}

When human perceive an image, color is an important part which they perceive. Color feature is one of the most widely used features for disease detection. HSV (Hue, Saturation and Value) is most widely used modal in which hue distinguishes color, Saturation is degree of purity of color in image and value describes the brightness or intensity.

\section{- Texture}

Texture feature most widely used for image classification and image retrieval. Currently most of the researchers targeting plant leaf texture as the most important feature in classifying plants [3].

\section{- Shape}

Shape feature is the visual feature used in plant disease detection. Shape representations can be categories in to two parts: Boundary based or Region based representation.

\section{E. Classification}

Classification is the challenging task in image processing technique which is based on the classifiers. The main purpose of the classification is to correctly predict the value of a designated discrete class variable, given a vector of predictors or attributes. In plant disease detection classification is done based on whether the image is infected or not. Numbers of methods are used as a classifier.

\section{Related Work}

Over the past years, different Disease detection techniques have been proposed.
Tejal et. al [1] Propose a system for disease identification and grading. They done their work on pomegranate leaf and fruit and detect bacterial blight disease. To remove the shadow, which causes during image acquisition, morphology technique has been used as pre-processing. For segmentation K-means clustering method has been used. After segmentation AT (Total Area of leaf or fruit) and AD (Total disease area) are calculated. Using AT and AD PI (percentinfection) is calculated, Using PI grade of the disease is determined. For disease identification they consider two characteristics as for the leaf they checked diseased spot on leaf is bordered by yellow margin if yes then it signifies that leaf is infected by bacterial blight and for the fruit first black spots are identified and if crack passing through that black spot it signifies that fruit is infected by bacterial blight. By using proposed system they achieve precise, accurate and acceptable result.

Revathi and Hemalatha [2] give a Homogeneous pixel counting technique for cotton disease detection (HPCCDD). By using canny and sobel edge detection homogenous techniques segmentation is done while proposed HPCCDD algorithm has been used for analysis and classification. By using proposed algorithm 98.1\% accuracy has been achieved. Gavhale et. al [3] proposed a method for citrus leaf disease detection. In pre-processing step image enhancement and color space conversion have been done. In feature extraction method GLCM texture feature and color texture feature are extracted while for classification SVM classifier has been used.

Monika et. al [4] Give a system for disease detection and fruit grading. For feature extraction three feature vectors have been used, namely, color, texture and morphology in which morphology give better result. For the classification artificial neural network has been used. For the fruit grading two methods are used spread of disease and automated calculation of mango weight. In spread of disease method percent infection is calculated by using $\mathrm{K}$-means clustering and in second method by using number of pixel weight is calculated and as per the weight quality of the fruit is decided.

Ratnasari et. al [5] proposed a system for sugarcane leaf disease detection. Proposed system has been verified only on three diseases, namely, rust spot, ring spot and yellow spot. Total 30 testing data are taken in which 9 images with rust disease, 7 images with ring spot disease, and 14 images with yellow spot disease. In feature extraction combination of color and texture feature has been used while for classification SVM classifier is used. In SVM classifier four kinds of kernel are tested, namely, linear, quadratic, radial basis function, and polynomial with $3^{\text {rd }}$ order in which linear kernel give better result than other. But because of limitation of segmentation method only $80 \%$ accuracy has been achieved.

Devi and Ginardi [6] proposed a system for identification of sugarcane rust disease. First normal and diseased images are collected and pre-processed. After that feature extraction has been done by using different feature extraction method in which individual and combination of different methods both are analyzed. The combination of texture and color feature 


\section{International Journal of Science and Research (IJSR) \\ ISSN (Online): 2319-7064}

Index Copernicus Value (2013): 6.14 | Impact Factor (2014): 5.611

gives a better result while for classification SVM classifier is used. In SVM classifier four kinds of kernel are tested, namely, linear, quadratic, radial basis function, and polynomial with $3^{\text {rd }}$ order in which polynomial with $3^{\text {rd }}$ order give a better result than other. $98.5 \%$ accuracy has been achieved.

Asho and Vinod [7] proposed a system for quality evaluation of fruits which is based on neural network. In proposed system test has been done on apple fruit. Total 65 data has been taken for testing in which 20 images are from healthy fruit and 45 images are form diseased fruit. $\mathrm{YCbCr}$ color space method has been used for the segmentation. Various features are extracted in feature extraction method. Probabilistic Neural Network has been used as a classifier.

Bashish et. al [8] proposed a framework for detection \& classification of leaf diseases and stem diseases. Solution of the system tested on five diseases which are: Early scorch, Cottony mold, ashen mold, late scorch, and tiny whiteness. First color transformation is conducted. Then, K-means clustering is used for segmentation. Color Co-occcurrence method has been used for feature extraction in which color and texture feature are considered. Neural network classifier is used for classification which is based on statistical classification.

Rastogi et. al [9] has performed an image processing and machine vision based technology for leaf disease detection and grading. First pre-processing has been done on leaf images then segmentation is applied by using K-means clustering and Euclidean distance technique. In feature extraction GLCM matrix is considered in which contrast, energy, homogeneity, and correlation have been calculated. Artificial neural network has been used for the classification. For grading percentage infection has been calculated by using total leaf area (AT) and diseased area (AD). After calculating the percentage infection grading has been applied by using fuzzy logic.

Narvear and Patil [10] offered novel algorithms which were based on image processing for grape leaf diseases detection. First pre-processing has been done in which RGB image is converted into HSV format. Then feature extraction method has been done by using SGDM method in which five features are calculated like energy, homogeneity, contrast, cluster prominence and cluster shade. Drawback of the proposed system is, they do not applied segmentation because of that powdery mildew and downy mildew are not well classified.

\section{Conclusion}

Plant Disease detection is very momentous and efficient research field. The paper purpose is to present an outline of established method for plant disease detection and study of recent growth. During survey it is identified that the major techniques for detection of plant diseases are: Neural network and SVM for classification and K-means clustering for segmentation. All these techniques are used to analyses the healthy and diseased plants leaves, fruits and stem. As per the review it is clear that these disease detection techniques have an ability to detect plant diseases but still there is some limitation. Therefore, there is scope of improvement in the existing research.

\section{References}

[1] Tejal Deshpande, Sharmila Sengupta, and K.S.Raghuvanshi, "Grading \& Identification of Disease in Pomegranate Leaf and Fruit," IJCSIT, vol. 5 (3), pp 4638-4645, 2014.

[2] P.Revathi and M.Hemalatha, "Classification of Cotton Leaf Spot Diseases Using Image Processing Edge Detection Techniques," IEEE International Conference on Emerging Trends in Science, Engineering and Technology (INCOSET), Tiruchirappalli, pp 169-173, 2012.

[3] Ms. Kiran R. Gavhale, Prof. Ujwalla Gawande, and Mr. Kamal O. Hajari, "Unhealthy Region of Citrus Leaf Detection using Image Processing Techniques," IEEE International Conference on Convergence of Technology (I2CT), Pune, pp 1-6, 2014.

[4] Monika Jhuria, Ashwani Kumar and Rushik esh Borse, "Image processing for smart farming detection of disease and fruit grading," IEEE $2^{\text {nd }}$ International Conference on Image Information Processing (ICIIP), Shimla, pp 521-526, 2013.

[5] Evy Kamilah Ratnasari, Mustika Mentari, Ratih Kartika Dewi and R. V. Hari Ginardi, "Sugarcane Leaf Disease Detection and Severity Estimation Based On Segmented Spots Image," IEEE International Conference on Information, Communication Technology and System (ICTS), Surabaya, pp 93-98, 2014.

[6] Ratih Kartika Dewi, and R. V. Hari Ginardi, "Feature Extraction for Identification of Sugarcane Rust Disease," IEEE International Conference on Information, Communication Technology and System (ICTS), Surabaya, pp 99-104, 2014.

[7] VaniAshok, and Dr.D.S.Vinod, "Automatic Quality Evaluation of Fruits Using Probabilistic Neural Network Approach," IEEE International Conference on Contemporary Computing and Informatics (IC3I), Mysore, pp 308-311, 2014.

[8] Dheeb Al Bashish, Malik Braik, and Sulieman BaniAhmad, "Feature Extraction for Identification of Sugarcane Rust Disease," IEEE International Conference on Signal and Image Processing (ICSIP), Chennai, pp 113-118, 2010.

[9] Aakanksha Rastogi, Ritika Arora and Shanu Sharma, "Leaf Disease Detection and Grading using Computer Vision Technology \&Fuzzy Logic," IEEE 2015 2nd International Conference on Signal Processing and Integrated Networks (SPIN), Noida, pp 500-505, 2015.

[10] Pradnya Narvekar, and Prof. S. N. Patil, "NOVEL ALGORITHM FOR GRAPE LEAF DISEASES DETECTION," International Journal of Engineering Research and General Science, vol.3, pp 1240-1244, 2015. 